\title{
Fluorescein angiography of the iris in anterior segment pigment dispersal syndrome
}

\author{
W E GILLIES AND C TANGAS \\ From the Glaucoma Investigation and Research Unit, Royal Victorian Eye and Ear Hospital, Melbourne, \\ Australia
}

SUMMARY The results are presented of fluorescein angiography of the iris in 11 patients with anterior segment pigment dispersal syndrome. These show a general hypovascularity of the iris with fine neovascularisation at the pupil margin and the peripupillary area. Hypoplasia of the iris stroma was also present in many cases. When the condition was virtually unilateral, the vascular changes were present though less marked in the relatively unaffected eye. It is postulated that the anterior segment pigment dispersal syndrome is secondary to a congenital mesodermal deficiency of the iris stroma with hypovascularity of the iris, which forms a poor support tissue for the pigment epithelium of the iris, resulting in shedding of pigment granules particularly in the region of the attachment of the dilator muscle to the pigment epithelium. The condition may be hereditary. Because of the hypovascularity the mesodermal hypoplasia may be progressive, but pigment release may diminish in later life with treatment, with consequent diminution of pupil activity.

The cause of the release of pigment granules from the pigment epithelium of the iris in the anterior segment pigment dispersal syndrome is of interest, as it is generally accepted that the deposition of the granules in the drainage angle causes the pigmentary glaucoma which usually accompanies this syndrome. The condition was considered by Perkins and Jay,' who drew attention to the numerous iris processes commonly seen in pigmentary glaucoma, which they considered to be due to a developmental defect of the outflow channels, with the deposition of pigment possibly contributing to obstruction of aqueous outflow but not being the primary cause.

Fine et al..$^{2}$ thought pigmentary glaucoma to be a developmental defect with idiopathic dysplasia of the iris epithelium and a distinct condition from chronic simple glaucoma. Kupfer et al. ${ }^{3}$ considered it to be a congenital or developmental abnormality especially affecting the inner and outer epithelial layers of the iris. Campbell ${ }^{4}$ considered the pigment release to be due to mechanical factors as the iris rubbed against the anterior packets of the ciliary zonules especially in myopic eyes.

In a prescient paper Lichter ${ }^{5}$ thought that an iris stromal defect, possibly vascular, may be the link

Correspondence to Dr W E Gillies, 82 Collins Strect, Melbourne, Victoria 3000, Australia. between the abnormalities of the drainage angle commonly seen in pigmentary glaucoma and the defects in the pigment epithelium of the iris. Olander

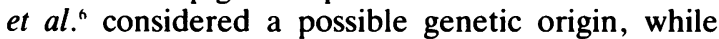
Scheie and Cameron' considered a multifactorial inheritance pattern. Chew and Deutman" reported one case of pigment dispersal syndrome and pigmented pattern dystrophy of the retinal pigment epithelium, though a recent series" ${ }^{9}$ of cases of pigmentary glaucoma showed no association with disorders of the pigment epithelium elsewhere in the eye.

To elucidate further the cause of the pigment release in this condition we have carried out iris fluorescein angiography on a number of cases, correlating the results with the clinical findings. This study has led us to the conclusion that anterior segment pigment dispersal syndrome is caused by a fault in the mesodermal support tissue to the iris and in particular a relative deficiency of the iris vasculature.

\section{Material and methods}

Fluorescein angiography of the iris was carried out on 11 patients with pigment dispersal syndrome. A Zeiss fundus camera was used as the light source, and a 


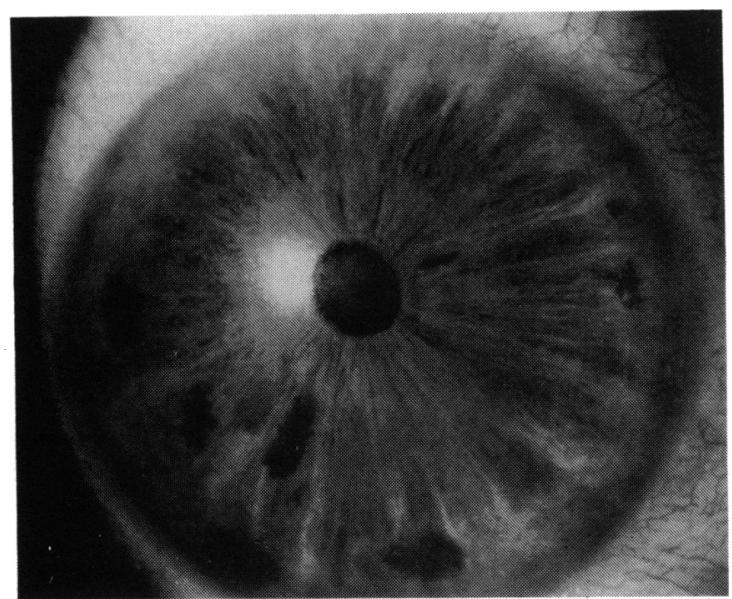

Fig. 1a Right eye of 55-year-old female, brown iris, showing the general thinning of the iris stroma, with a very open structure giving the appearance of a loose sponge work, the pigment epithelium being visible through the interstices of the stroma. The pupil is constricted by miotic drops.

Nikon F3 camera was used to take the photographs with a $105 \mathrm{~mm}$ Macro lens, a PN -1 tube, and CU close-up lenses. The F stop was $\mathrm{f} 5.6$ for the first and $\mathrm{f} 8$ for the second run. The fluorescein dosage was $5 \mathrm{ml}$ $25 \%$ for the first run and $3 \mathrm{ml} 25 \%$ for the second run. Tri-X film was processed with Microdol X stock solution developed at $22^{\circ} \mathrm{C}$ for 14 minutes to obtain the negatives.

On gonioscopy all these patients had a heavy deposition of pigment in the trabecular tissue of the drainage angle. In addition to this there was other evidence of pigment dispersal, namely, reflux of light through the pigment epithelium of the iris in all cases, Krukenberg spindles in all cases, and pigment deposition at the attachment of the zonule to the lens in five cases.

Corneal endothelial cell counts were carried out on six patients, and all cases were examined with the slitlamp for diffuse atrophy or hypoplasia of the iris stroma. This was assessed on thinning and loss of the normal structure of the iris stroma, so that the normal radial structure of the iris was less apparent, and it was possible to see some detail of the pigment epithelium through the thinned stroma.

The patients comprised seven males and four females aged 26 to 73 , average age 48.7 years, range $26-73$, SD $12 \cdot 1$ years. Most had light coloured irides, only two having a brown iris. Three patients were under the age of 40 and seven were under 50 .

\section{Results}

Evidence of atrophy of the iris stroma was found on

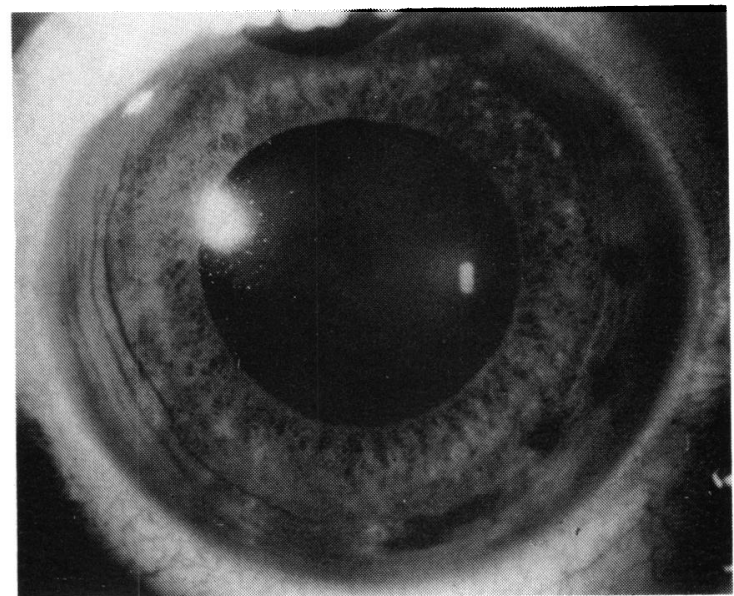

Fig. 1b Left eye of same patient as in Fig. Ia with similarly open stromal meshwork. The eye has previously had a drainage operation and the pupil is mobile and semidilated, though no drops are being used.

biomicroscopy in six patients. It consisted of loss of the normal iris architecture and a thinning of the iris stroma so that the pigment epithelium could be seen easily through the stroma. These patients often had semidilated pupils which did not contract fully with miotics, perhaps owing to a general loss of iris tissue and an effect on the iris sphincter muscle. (Figs. 1,2).

In four patients the pigment dispersal was much more marked in one eye than the other, in which it seemed slight though present to a limited degree. One such patient had normal intraocular pressure in each eye. In the other three the more affected eye

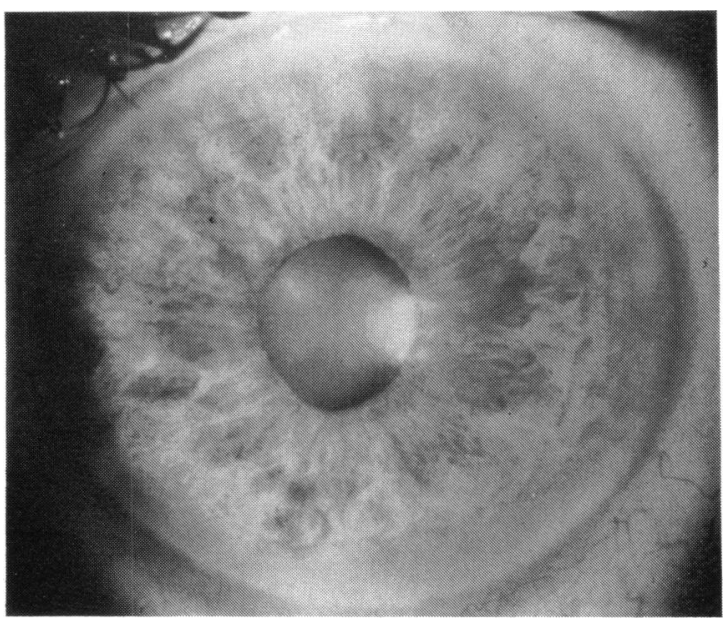

Fig. 2 Right eye of a 73-year-old male, light grey iris with pigment deposition in the stroma, which shows diffuse atrophy. The pupil is not completely miotic in spite of strong miotic drops. 


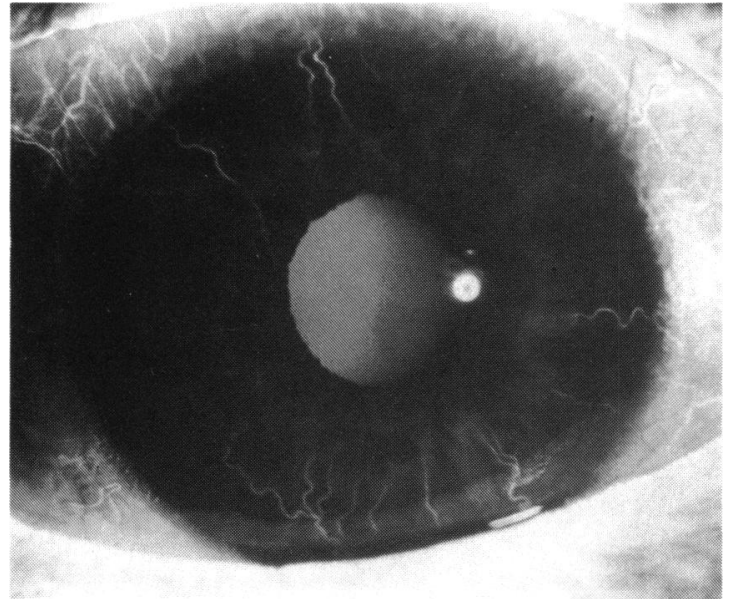

Fig. 3a Right eye of a 58-year-old male at 25.7 seconds; arterial phase has just begun. Note extreme paucity of vessels in the upper and temporal quadrants of the iris.

had the higher pressure, the fellow eyes being within normal limits.

On iris angiography all eyes showed vascular hypoperfusion of the iris with scant, attenuated iris vessels. In four eyes no radial arteriole was seen in one quadrant of the iris or only one to three arterioles were present to supply adjoining quadrants (Figs. 3 ae). In all the other eyes the number of arteries was greatly reduced and their calibre attenuated (Figs. 4, 5). Appearance of the dye in the arterioles was at an average of 25 seconds (range 21-32 s, SD 2.3 s). Appearance of dye in the veins seemed to be delayed for an average of $10 \cdot 1$ seconds later (range $7-15 \mathrm{~s}, \mathrm{SD}$ $2 \cdot 3 \mathrm{~s})$.

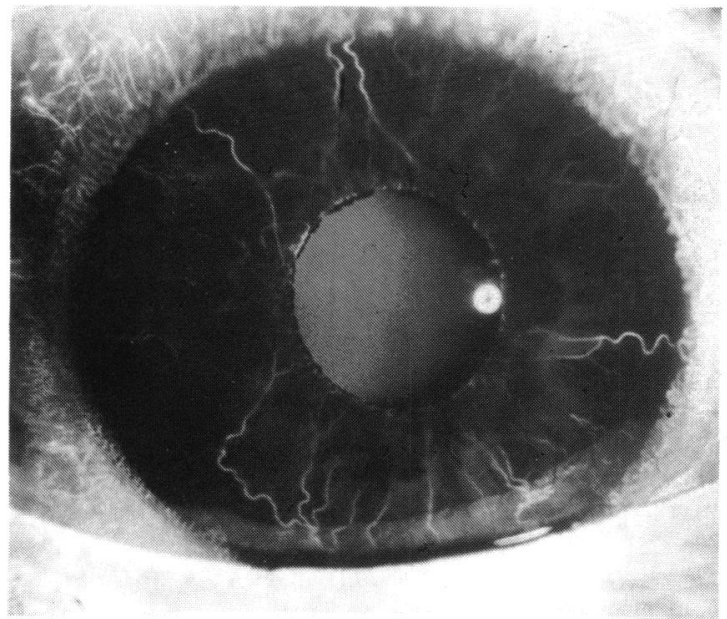

Fig. 3c Dye has now reached tufts at the pupil margin. Arterial phase at $32 \cdot 2$ seconds.

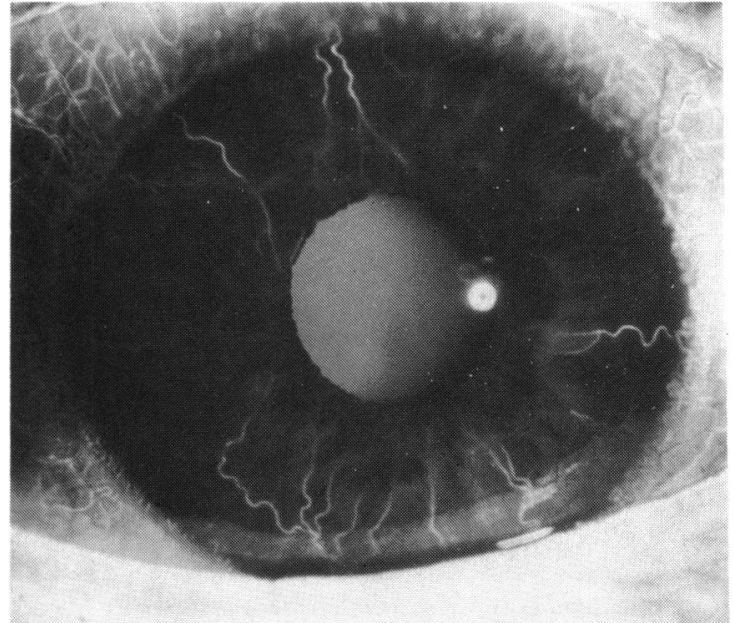

Fig. 3b Same eye at 28.9 seconds; the arterial phase is well established and the lack of arterioles previously noted persists.

All eyes showed leakage of dye from fine peripupillary neovascularisation, with leaks also occurring from neovascular tufts at the pupil margin. This leakage from peripupillary vessels and from the pupil margin was copious in late pictures, strongly suggesting that this was neovascularisation and not merely the unmasking of a normal capillary network by the iris atrophy (Figs. 3e, 3f).

All but two patients showed aberrant vessels and loops in the peripheral iris stroma, with some leak from neovascular tufts in the peripheral stroma. These findings were present in both the younger and the older patients. The two patients with a brown iris showed findings similar to but less marked than those

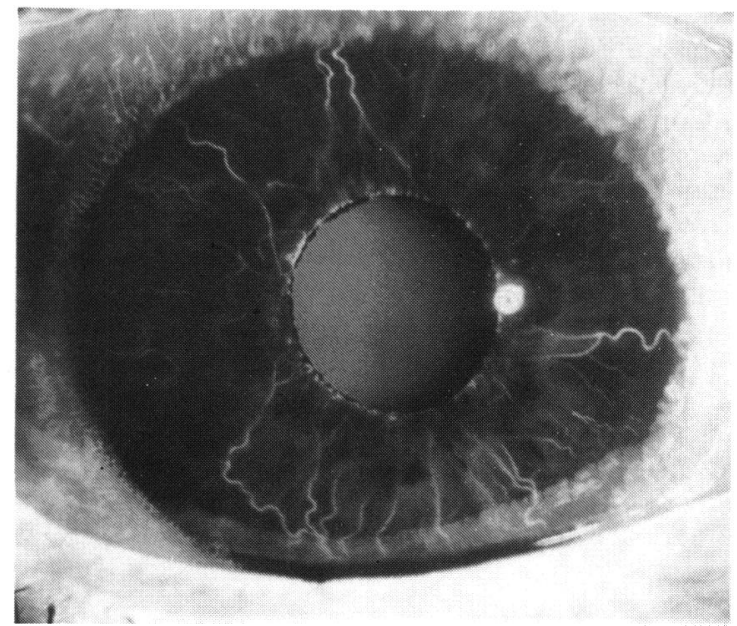

Fig. 3d At 34.8 seconds the venous phase is just beginning, and some leak is occurring at the pupil margin. 


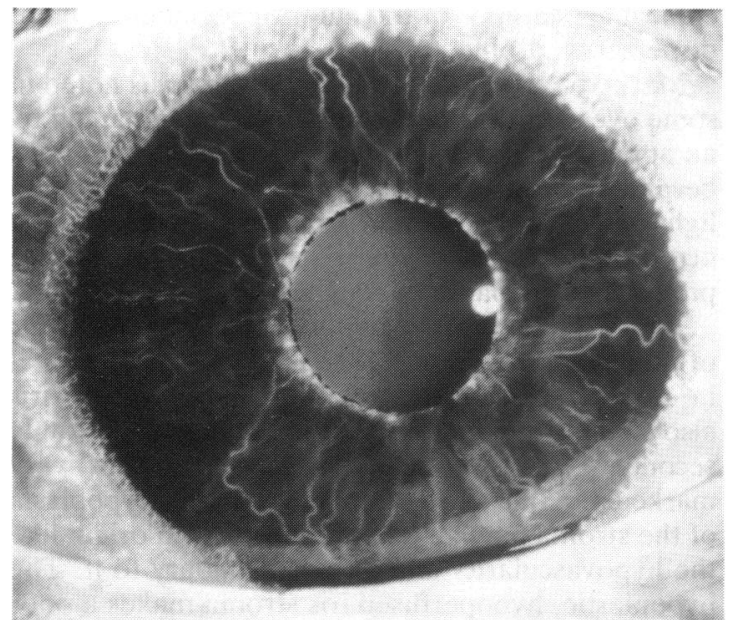

Fig. 3e At $43 \cdot 7$ seconds the venous phase is well established, and there is considerable leak from the pupil margin.

with a blue iris, the masking of the vascular pattern which occurs with a brown iris being diminished by the iris atrophy (Figs. 4a, 4b). In the four patients in whom pigment dispersal was more marked in one eye than in the fellow eye the findings on iris angiography were similar to those from the other eyes in the group. Though they were less marked in the less affected eye in these patients, the vascular changes were still present (Figs. 3a-f).

Corneal endothelial cell counts were performed on six of the patients and ranged from 2000 to 2675 cells $/ \mathrm{mm}^{2}$ with some pleomorphism in three patients,

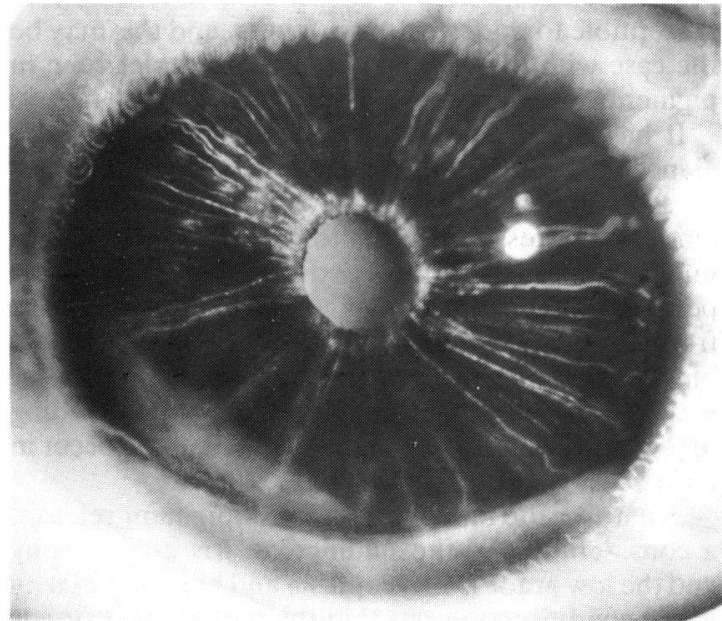

Fig. 4a . Iris angiography at $42 \cdot 2$ s of same eye as in Fig. Ia, showing relatively sparse iris radial vessels with early pupillary and peripupillary neovascularisation.

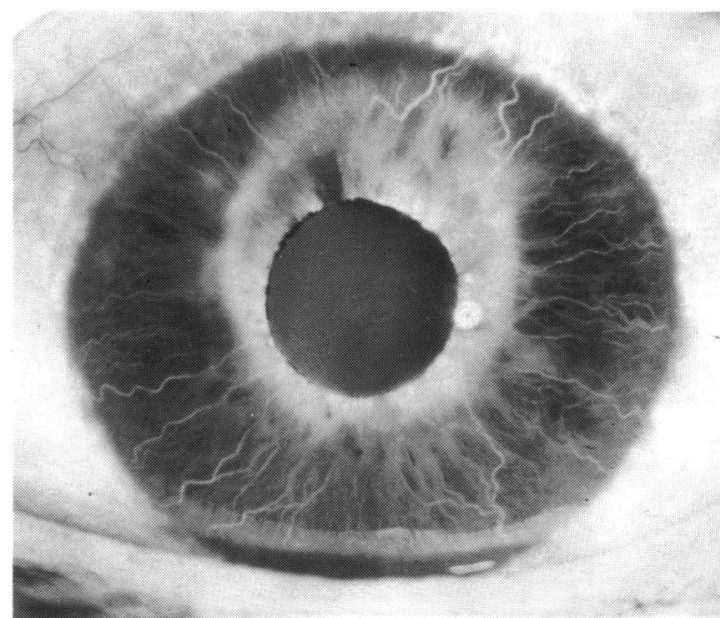

Fig. 3f The left eye of the same patient at 58.5 seconds in the second run. This fellow eye showed little evidence of pigment dispersal, but there was relative hypoperfusion of the iris, and a late heavy leakage of dye from pupillary and peripupillary vessels is evident with a 'smoke stack' effect.

suggesting some interference with endothelial cell function in these unoperated eyes.

\section{Discussion}

The normal iris angiogram 10 "shows radially running arterioles which course from the iris root towards the pupil, tapering a little in calibre and following a slightly tortuous course. Few collateral branches are given off, and near the pupil the arterioles break up

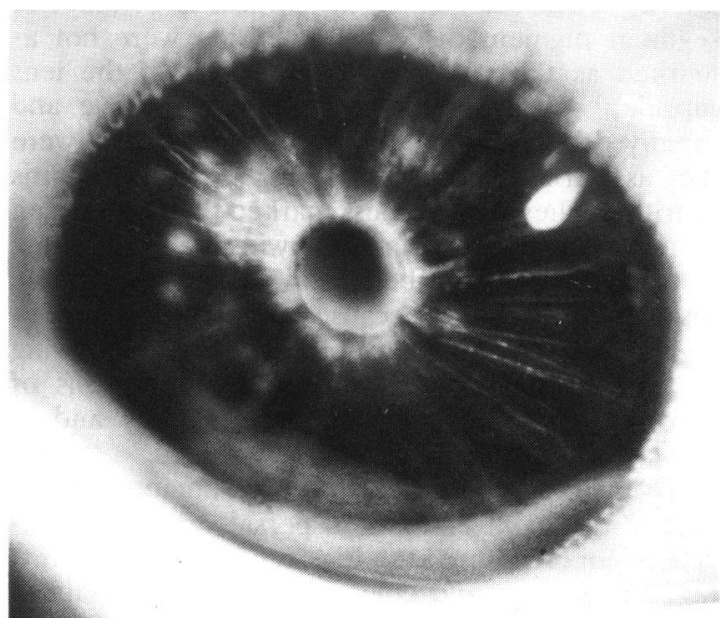

Fig. 4b The same patient at $71 \cdot 8$ showing much heavier leakage from the pupillary and peripupillary neovascularisation. There is also some leakage of dye in the more periperhal iris stroma. 


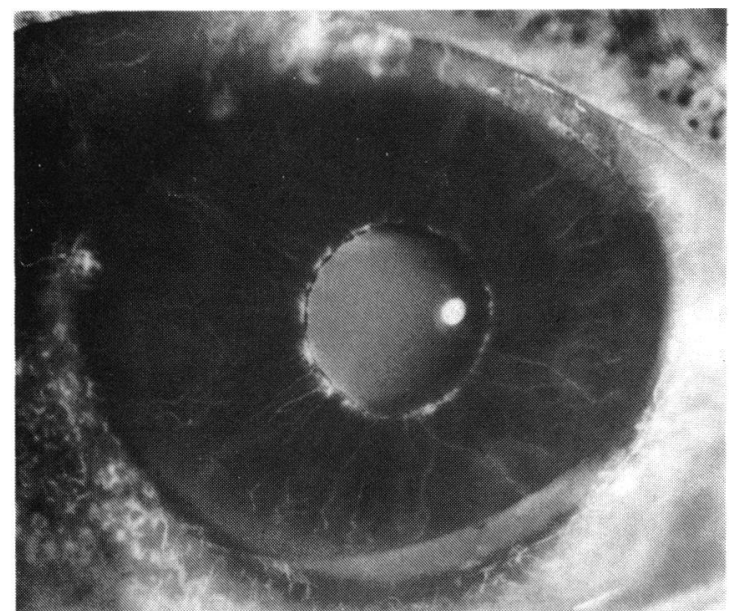

Fig. 5 Iris angiography at 61.5 s of same patient as in Fig. 2, showing the poor and attenuated vasculature with leaking neovascularisation in the pupillary and peripupillary area.

into fine vessels which interlace to form a fine peripupillary network, and arteriovenous bridges may be seen at the pupil border. The arterioles are evenly distributed in all the quadrants of the iris. Veins run a similar radial course, are more numerous and deeper. The arterioles fill 9-20 or more seconds after dye is injected in the arm and the veins fill about 4 seconds after the arterioles. No leakage is seen in young patients, but some patients over the age of 50 will show small tufts of fluorescein leakage at the pupil margin, so that it may or may not be pathological in patients over that age.

The neovascular changes in the iris in anterior segment pigment dispersal syndrome were not as marked as those in pseudoexfoliation of the lens capsule, ${ }^{12}$ which seems to be a more active and rapidly developing pathological process. Nor were they as marked as those occurring in chronic cyclitis or iritis. ${ }^{13}$ They were far less marked then those seen in neovascular glaucoma following central retinal vein occlusion or in diabetic neovascularisation of the iris. ${ }^{14}$ This is probably because neovascular change in pigmentary glaucoma develops very gradually owing to a congenital defect, while the other forms of neovascular change develop more rapidly and in neovascular glaucoma very rapidly indeed.

We believe these findings on iris angiography in anterior segment pigment dispersal syndrome indicate hypoperfusion of the iris even in young patients together with a mild degree of neovascularisation.

These changes are present, though less marked, in the relatively unaffected eye when the pigment dispersal is virtually unilateral. This suggests that the mesodermal and vascular changes precede the clinical appearance of the anterior segment pigment dispersal syndrome.

Marked hypoplasia of the iris stroma was noted in some eyes, and this was sometimes accompanied by an apparent shrinkage of the iris, so that the pupils became wide and semidilated, though still reacting to light and accommodation. Ectropion uveae did not occur, but atrophy of the iris stroma seemed to be progressive in patients watched over a long time.

We postulate that the hypovascularity of the iris is of very long standing, probably being congenital in origin, so that the other changes in the iris also develop slowly. Neovascular changes, probably secondary to the hypovascularity, are not very marked and their development is subtle. Hypoplasia of the stroma may be partly congenital in origin like the hypovascularity and partly secondary to it. The hypoplastic, hypoperfused iris stroma makes a poor support tissue for the iris pigment epithelium, which is therefore susceptible to degenerative change and release of its pigment granules.

We further suggest that the pigment release in anterior segment pigment dispersal syndrome is due to the congenital mesodermal deficiency of the iris stroma. The ultimate cause of the pigment release may be mechanical, as suggested by Campbell, ${ }^{4}$ by rubbing of the iris against the ciliary packets, especially in myopic eyes, but in a recent series" most eyes were not myopic and most of the myopia was less than $2 \cdot 0$ dioptres.

It is likely that the pigment release from the iris epithelium and its deposition elsewhere in the anterior segment, especially in the trabecular meshwork, cause obstruction to aqueous outflow and rise in intraocular pressure. Some eyes may be more susceptible to this effect than others, and this may be the case with the well marked iris processes seen in pigmentary glaucoma.

It has been noted that patients with pigmentary glaucoma are often tense, and attacks of high rise in pressure may be precipitated by physical exertion or emotional stress. ${ }^{15}$ The dilator muscle of the iris is under sympathetic control and is inserted into the iris peripherally in the region of greatest pigment release from the iris. Perhaps this is the final mechanism of pigment release, and, as iris mobility decreases with age and treatment, there is diminished shedding of pigment granules, which has been noted to occur in time. ${ }^{16}$

Whatever the final mechanism of pigment release, a consideration of the findings on iris angiography and the low grade iris hypoplasia and hypovascularity in the condition suggests that the underlying cause is a congenital deficiency of the mesodermal support tissue of the iris, which occurs early in development and is probably congenital. 
Our thanks are due to the Photography Department of the Royal Victorian Eye and Ear Hospital, particularly Mrs G Grant, to Miss J Quilter of the Ronald Lowe Library Royal Victorian Eye and Ear Hospital, and to Miss C Atkinson for secretarial assistance.

This work was carried out under Research Project 20 of the Royal Victorian Eye and Ear Hospital.

\section{References}

1 Perkins ES, Jay BS. Pigmentary glaucoma. Trans Ophthalmol Soc UK 1960; 80: 153-67.

2 Fine BS, Yanoff M, Scheie RG. Pigmentary 'glaucoma'. Opthalmology (Rochester) 1974; 78: OP314-OP325.

3 Kupfer C, Kuwabara T, Kaiser Kupfer M. The histopathology of pigmentary dispersion syndrome with glaucoma. Am J Ophthalmol 1975; 80: 857-62.

4 Campbell DG. Pigmentary dispersion and glaucoma. Arch Ophthalmol 1979; 97: 1667-72.

5 Lichter PR. Pigmentary glaucoma-current concepts. Ophthalmology (Rochester) 1974; 78: OP309-OP313.

6 Olander KW, Mandelkorn, Hoffman ME, Zimmerman. Pigment dispersion syndrome and open angle glaucoma. Ann Ophthalmol 1982; 14: 809-10.

7 Scheie HG, Cameron JD. Pigment dispersion syndrome: a clinical study. Br J Ophthalmol 1981; 65: 264-9.
8 Chew EY, Deutman AF. Pigment dispersion syndrome and pigmented pattern dystrophy of retinal pigment epithelium $B r J$ Ophthalmol 1983; 67: 538-41.

9 Gillies WE. Pigmentary glaucoma-a clinical review of anterior segment dispersal syndrome. Aust J Ophthalmol in press.

10 Vannas A. Fluorescein angiography of the vessels of the iris in pseudoexfoliation of the lens capsule, capsular glaucoma and some other forms of glaucoma. Acta Ophthalmol (Kbh) 1969; 105 (suppl): 1051-75.

11 Kottow MH. Anterior segment fluorescein angiography. Baltimore: Williams and Wilkins, 1978: 35-55.

12 Brooks AM, Gillies WE. Fluorescein angiography and fluorophotometry of the iris in pseudoexfoliation of the lens capsule. $\mathrm{Br}$ J Ophthalmol 1983; 67: 249-54.

13 Kottow MH. Anterior segment fluorescein angiography. Baltimore: Williams and Wilkins, 1978: 111-28.

14 Kottow MH. Anterior segment fluorescein angiography. Baltimore: Williams and Wilkins, 1978: 129-51.

15 Schenker HI, Lunt MH, Kels B, Podos SM. Exercise-induced increase in intraocular pressure in the pigmentary dispersion syndrome. Am J Ophthalmol 1980; 89: 598-600.

16 Ritch R. Pigmentary glaucoma: a self-limited entity. Ann Ophthalmol 1983; 15: 115-6.

Accepted for publication 15 August 1985. 